\title{
The Role of White Matter Damage in the Risk of Periprocedural Diffusion-Weighted Lesions after Carotid Artery Stenting
}

\author{
Paola Maggio ${ }^{a}$ Claudia Altamurab ${ }^{b}$ Domenico Lupoi ${ }^{d}$ Matteo Paolucci ${ }^{b}$ \\ Riccardo Altavilla ${ }^{b}$ Francesco Tibuzzic ${ }^{c}$ Francesco Passarellic

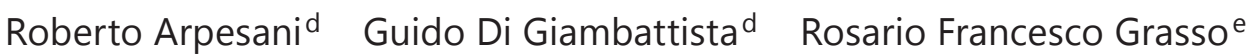 \\ Giacomo Luppi $^{\mathrm{e}}$ Fabrizio Fiacco $^{\mathrm{a}}$ Mauro Silvestrini $^{f}$ \\ Patrizio Pasqualetti ${ }^{9}$ Fabrizio Vernieri ${ }^{b}$

\begin{abstract}
${ }^{a}$ Neurology Unit, ASST Bergamo Est, Azienda Ospedaliera Bolognini, Seriate, ${ }^{b}$ Headache and Neurosonology Unit, Neurology, Policlinico Universitario Campus Bio-Medico,

cNeurology Unit, S. Giovanni Calibita, Fatebenefratelli Hospital, dRadiology Unit, S. Giovanni Calibita, Fatebenefratelli Hospital, and ${ }^{\mathrm{e}}$ Radiology Unit, Policlinico Universitario Campus Bio-Medico, Rome, ${ }^{f}$ Neurological Clinic, Università Politecnica delle Marche, Ancona, and 9SESMIT, Fatebenefratelli Foundation for Health Research and Education, Fatebenefratelli Hospital at Isola Tiberina, Rome, Italy
\end{abstract}

\section{Keywords}

Carotid artery stenting - Diffusion-weighted images - White matter hyperintensities

\begin{abstract}
Background: White matter hyperintensities (WMH) are a common finding in aged individuals affected by carotid artery disease and are a risk factor for first-ever and recurrent stroke. We investigated if white matter damage increases the risk of brain microembolism during carotid artery stenting (CAS), as evaluated by the appearance of new areas of restricted diffusion on diffusion-weighted images (DWI). Methods: We evaluated 47 patients with severe internal carotid artery (ICA) stenosis undergoing CAS, comparing preprocedural clinical, ultrasound and radiological characteristics. WMH volume was computed on FLAIR images before CAS. After CAS, the DWI scan was looked over for areas of restricted diffusion (DWI lesions). A first univariate analysis was adopted to compare groups according to the occurrence of DWI lesions. Then, the variable DWI lesion was modelled by means of a logistic regression model. Results: Seventeen patients developed at least 1 DWI lesion after CAS. Compared with nonDWI, DWI patients were more commonly treated in the left ICA $(p=0.007)$ and had a more severe WMH damage ( $p=0.027$ ). Indeed, the risk of a DWI lesion was higher in left versus right stenosis $(\mathrm{OR}=9.0,95 \% \mathrm{CI} 1.9-42.7, p=0.005)$ and increased for each log-unit of WMH lesion load (OR $=7.05,95 \%$ CI 1.07-46.49, $p=0.042)$. A WMH lesion load of at least $5.25 \mathrm{~cm}^{3}$
\end{abstract}



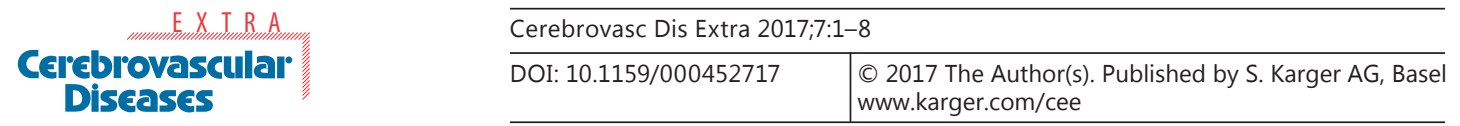

Maggio et al.: The Role of White Matter Damage in the Risk of Periprocedural

Diffusion-Weighted Lesions after Carotid Artery Stenting

had a $50 \%$ probability of occurrence of a new DWI lesion. Conclusions: Treated side and preexisting white matter damage are risk conditions for brain microembolism during CAS. This should be taken into account to optimize severe carotid artery disease management.

(C) 2017 The Author(s)

Published by S. Karger AG, Basel

\section{Introduction}

Carotid artery stenting (CAS) is a procedure associated with high risk of brain microembolism, usually detected as areas of restricted diffusion on magnetic resonance (MR) diffusionweighted images (DWI). Although most CAS-related DWI lesions do not cause an obvious neurological deficit, they are demonstrated to impact cognitive function [1] and may represent a surrogate parameter for optimizing diagnostic and therapeutic vascular procedures [2].

Advanced age, plaque characteristics and procedures without emboli protection devices have been advocated as risk factors for new DWI lesions after CAS, even though with conflicting results [3-9].

Brain white matter hyperintensities (WMH) are a common finding in aged individuals affected by carotid artery disease [10]. Although their pathogenesis is largely unknown, a hemodynamic contribution has been supposed $[11,12]$. Different studies have shown that a reduced basal cerebral blood flow [13] as well as a poor cerebrovascular response to acetazolamide or hypercapnia are related to white matter changes [14-16].

Moreover, WMH areas present an impaired hemodynamic status, making these areas more likely to be exposed to transient ischemia, which may induce myelin rarefaction [17]. Furthermore, WMH are a recognized risk factor for first-ever and recurrent stroke in population-based studies [18].

We hypothesized that patients with a severe basal WMH lesion load could be more prone to develop DWI lesions during a procedure at high risk for distal embolization, such as CAS. To test this hypothesis, we investigated baseline clinical and radiological characteristics affecting the occurrence of periprocedural DWI lesions in our cohort of patients treated with CAS for severe internal carotid artery disease.

\section{Methods}

We retrospectively evaluated 47 patients (mean age 73 years, SD 8; 14 female) with severe (i.e., greater than 70\%) carotid artery stenosis, who underwent carotid angioplasty with stent placement at the Interventional Radiology Unit of our institutions from January 2007 to February 2013. Patient enrollment, and clinical, ultrasound and MR evaluations were performed as already described [1].

In summary, plaque characteristics and degree of stenosis were evaluated by ultrasound of neck vessels and confirmed by computed tomographic angiography. Intracranial vessels were examined with transcranial color-coded sonography and MR angiography to exclude significant stenosis of the large intracranial arteries.

All patients underwent electrocardiography and cardiological and neurological examination on admission. Brain MR imaging was performed the day before CAS. No patient had possible or probable embolizing cardiopathy. The day after the procedure, clinical assessment, brain MR imaging, and color-coded duplex sonography of the neck vessels were repeated. In addition, all patients underwent continuous electrocardiographic monitoring in the first 48 $\mathrm{h}$ following CAS to detect possible new-onset arrhythmias. 

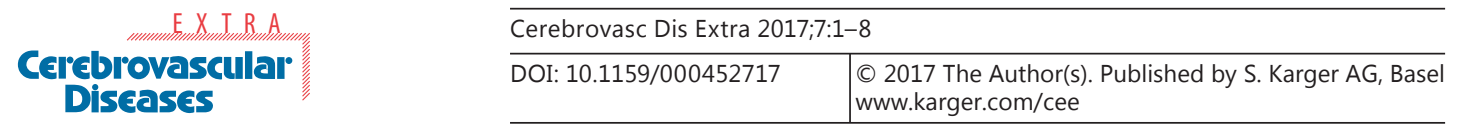

Maggio et al.: The Role of White Matter Damage in the Risk of Periprocedural Diffusion-Weighted Lesions after Carotid Artery Stenting

Therapy with ASA $100 \mathrm{mg}$ plus clopidogrel $75 \mathrm{mg}$ as tablets once a day was initiated at least 5 days before CAS in all patients. Clopidogrel was continued for 6 months after CAS and ASA was administered indefinitely. Each patient received the best medical therapy to control vascular risk factors. The institutional review committee approved the study. All the patients gave their informed written consent.

\section{Stent Placement}

Every CAS procedure in the present study was performed under local anesthesia in a dedicated operating room equipped for endovascular procedures by the same 2 experienced radiologists. A femoral access was used to introduce a guidewire up to the origin of the internal carotid artery (ICA). Carotid and cerebral angiography was then performed to confirm the level and the degree of arterial narrowing. The guidewire was then manipulated across the stenosis and a balloon was passed over the device to lie across the stenosis, where it was inflated manually. Thereafter a deployment of the stent into the area of narrowing was done. At the end of the procedure, an angiography was performed to assess the result. Either distal or proximal cerebral protection devices were used.

\section{DWI Lesions and WMH Assessment}

MR imaging analysis was conducted by an investigator blinded to clinical patients' data (D.L.). As previously described [1], MR imaging was performed with an Achieva 1.5-T scanner (Philips Medical Systems, Best, the Netherlands) and an 8-channel head phased-array coil with parallel imaging capabilities (SENSE). The following brain MR sequences were collected before and after CAS: axial plane T1-weighted spin echo $(\mathrm{TR}=550, \mathrm{TE}=15$, slice thickness $=$ $3 \mathrm{~mm}$, interslice gap $=0$ ), FLAIR ( TR $=8,000, \mathrm{TE}=125, \mathrm{TI}=2,500$, slice thickness $=3 \mathrm{~mm}$, interslice gap $=0)$, and DWI (TR $=3,220, \mathrm{TE}=88, b$ value $=1,000$, slice thickness $=5 \mathrm{~mm}$, interslice gap $=1 \mathrm{~mm}$ ) with apparent diffusion coefficient.

Total WMH volume was computed on FLAIR axial images before CAS, after having segmented lesional regions of interest through a semi-automated local thresholding approach (Osirix ${ }^{\circledR}$ software V.3.9.4) [19]. After CAS, the DWI scan was looked over for areas of restricted diffusion (DWI lesions), which were classified according to anatomical localization and side of the treated artery.

\section{Statistical Analysis}

WMH distributions were not gaussian (Shapiro-Wilks, $p<0.001$ ) and markedly improved the fit after a logarithmic transformation (ratio skewness/SE[skewness] decreased from 3.7 [ $p<0.001]$ to $1.1[p=0.28]$ ). A first analysis including baseline parameters was adopted to compare groups based on the occurrence of postprocedural DWI lesion and served as the basis for a multivariate logistic regression model. Continuous variables were compared using the Mann-Whitney U test or the Student $t$ test, as appropriate. The distributions of the categorical variable were compared using the $\chi^{2}$ test.

The number of DWI lesions after CAS was put in relation to potential covariates assuming a negative binomial distribution. The variable DWI lesion was also dichotomized $(0=$ no DWI lesion; 1 = at least 1 DWI lesion) and modelled by means of a logistic regression model. Since the number of patients in the smaller category (i.e., occurrence of at least 1 DWI lesion) was 17 , our sample was not large enough to reliably assess the effect of several variables. Thus, we entered in the multivariate model only the variables that showed a significant univariate association with the outcome. Antithrombotic drugs were not included in the multivariate analysis as we prescribed both ASA and clopidogrel to all patients. Data analysis was performed with SPSS ${ }^{\circledR}$ (version 19) for Windows. 

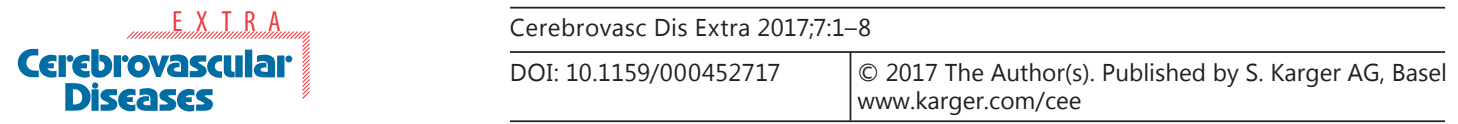

Maggio et al.: The Role of White Matter Damage in the Risk of Periprocedural

Diffusion-Weighted Lesions after Carotid Artery Stenting

Table 1. Comparison of clinical, ultrasound and MR imaging data between DWI and non-DWI groups

\begin{tabular}{|c|c|c|c|c|}
\hline Characteristics & $\begin{array}{l}\text { All } \\
(n=47)\end{array}$ & $\begin{array}{l}\text { DWI- } \\
(n=30)\end{array}$ & $\begin{array}{l}\text { DWI+ } \\
(n=17)\end{array}$ & $p$ value \\
\hline \multicolumn{5}{|l|}{ Clinical data } \\
\hline Age, years (SD) & $73(8)$ & $75(7.2)$ & $72(8.3)$ & 0.248 \\
\hline Gender (female), $n(\%)$ & $14(30)$ & $10(33)$ & $4(24)$ & 0.480 \\
\hline Hypertension, $n(\%)$ & $39(83)$ & $25(83)$ & $14(82)$ & 0.932 \\
\hline Diabetes, $n(\%)$ & $20(43)$ & $11(37)$ & $9(53)$ & 0.278 \\
\hline Hypercholesterolemia, $n(\%)$ & $37(79)$ & $26(87)$ & $11(65)$ & 0.077 \\
\hline Smoking habit, $n(\%)$ & $18(38)$ & $11(37)$ & $7(41)$ & 0.760 \\
\hline Ipsilateral previous stroke/TIA ${ }^{\mathrm{a}}, n(\%)$ & $11(23)$ & 5 (17) & $6(35)$ & 0.147 \\
\hline Statin therapy, $n(\%)$ & $29(62)$ & $20(67)$ & $9(53)$ & 0.352 \\
\hline \multicolumn{5}{|l|}{ Ultrasound and procedure-related findings } \\
\hline Degree of stenosis, \% (SD) & $77.9(7.9)$ & $78.2(8.3)$ & $77.4(7.5)$ & 0.739 \\
\hline Side of stenosis (left), $n(\%)$ & $21(45)$ & $9(30)$ & $12(71)$ & 0.007 \\
\hline Ulcerated plaque, $n(\%)$ & 8 (17) & 7 (23) & $1(5.8)$ & 0.126 \\
\hline Embolic protection (distal), $n(\%)$ & $43(91)$ & $26(60)$ & $17(39)$ & 0.115 \\
\hline \multicolumn{5}{|l|}{ MR imaging analysis } \\
\hline WMH, $\mathrm{cm}^{3}(\mathrm{SD})$ & $3.9(4.8)$ & $2.4(3.2)$ & $6.3(6.0)$ & $0.027^{b}$ \\
\hline
\end{tabular}

TIA, transient ischemic attack. Figures in bold are significant.

${ }^{a}$ More than 6 months before procedure. ${ }^{\mathrm{b}} t$ test applied after log-transformation.

\section{Results}

Table 1 displays preprocedural clinical characteristics, ultrasound, MR imaging and procedure-related data of the patients enrolled and a comparison between the DWI-positive (DWI+) and DWI-negative (DWI-) groups.

Preprocedural DWI were negative in all patients. All patients had a complete recanalization after the procedure. No major complications were observed either during the intervention or during the follow-up period. One patient reported transient (lasting less than $2 \mathrm{~h}$ ) left upper limb weakness early after CAS and reported a new DWI lesion on the right precentral gyrus. No other patient presented symptoms suggestive of stroke. Seventeen (36\%) out of 47 patients developed at least 1 new DWI lesion after CAS (DWI+ group), without persistent clinical deterioration (Fig. 1). In the DWI+ group, we found a total of 71 new lesions. Fiftyseven $(80 \%)$ were ipsilateral to the treated artery and 14 were contralateral. Forty DWI lesions (56\%) were located in the frontal lobe, 5 (7\%) in temporal, $12(16 \%)$ in parietal, 7 $(10 \%)$ in occipital, and $7(10 \%)$ in basal nuclei. Eight (17\%) patients had an ulcerated carotid plaque and distal embolic protection was adopted in 91\% of cases (43 patients).

DWI+ patients were more commonly treated in the left ICA compared with DWI- patients $(p=0.007)$. Moreover, DWI + patients had a more severe preprocedural WMH lesion load $(p=0.027)$. No differences were found with respect to clinical data, plaque ulceration or procedure-related findings $(p>0.05)$. History of ipsilateral stroke or transient ischemic attack did not affect the outcome.

When both laterality of ICA and WMH lesion load had been entered in the logistic model, the occurrence of at least 1 new DWI lesion was confirmed related to both variables. The risk of DWI lesions was higher in the left- versus right-side stenosis (OR $=9.0,95 \%$ CI 1.9-42.7, $p=0.005)$ and increased for each log unit of WMH lesion load (OR $=7.05,95 \%$ CI 1.07-46.49, $p=0.042$ ). Regardless of the side of the stenosis, we observed that a patient with a WMH lesion load of $5.25 \mathrm{~cm}^{3}$ has a $50 \%$ probability of having new DWI lesions after CAS. It should 


\section{Cerebrovascular \\ Diseases}

Table 2. Stepwise logistic regression analysis with postprocedural DWI lesion occurrence as dependent variable

\begin{tabular}{l}
\hline Cerebrovasc Dis Extra 2017;7:1-8 \\
\hline DOI: 10.1159/000452717
\end{tabular}

Maggio et al.: The Role of White Matter Damage in the Risk of Periprocedural Diffusion-Weighted Lesions after Carotid Artery Stenting

\begin{tabular}{lrcl}
\hline Independent variable & \multicolumn{1}{c}{ OR } & \multicolumn{1}{c}{ CI } & $p$ \\
\hline Age & 1.15 & $0.99-1.33$ & 0.054 \\
Left-side stenosis & 17.12 & $2.070-141.64$ & 0.008 \\
WMH log & 8.97 & $1.08-74.44$ & 0.042 \\
\hline
\end{tabular}
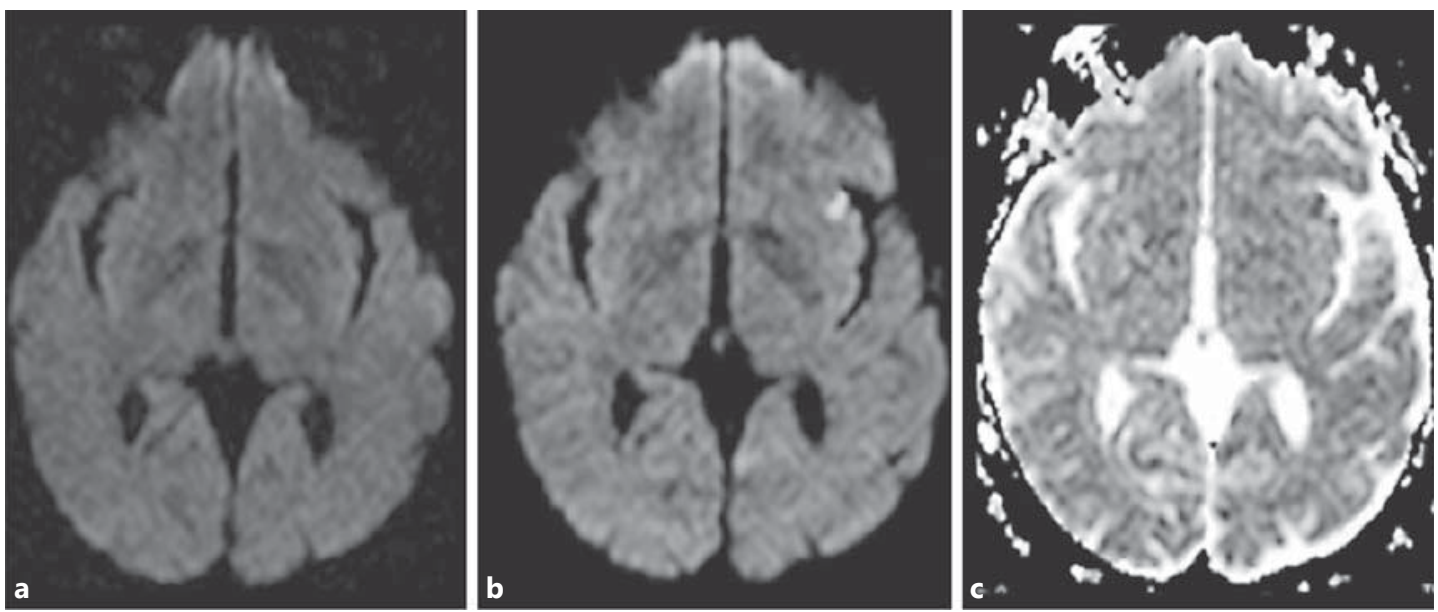

Fig. 1. Example of an MR imaging study. a Preprocedural DWI scan, negative for DWI restriction areas. b Postprocedural DWI scan, showing left insular DWI restriction. c Postprocedural apparent diffusion coefficient map, confirming a new ischemic lesion on the left insula.

be noted that when age was entered as a "forced" term to remove the plausible aging effect, the effects of side and WMH remained similar (Table 2).

\section{Discussion}

In the present study, we retrospectively investigated which factors were most related to the DWI lesions that occurred during the CAS procedure in patients with ICA severe stenosis. Our analysis showed that the treated side (with left > right) and preexisting white matter damage severity were the most relevant risk conditions, also after age correction.

Distal embolization during carotid endovascular procedures could theoretically be related entirely to mechanical factors, i.e., the shear stress applied to the atheroma during balloon inflation or stent deployment, inducing plaque fragmentation and debris migration from the treated artery, or from the aortic arch or common carotid arteries before stent placement. According to this hypothesis, the anatomical features of vessels and plaque morphology should be the main determinants of the risk of periprocedural stroke or DWIrestricted lesions after CAS. Indeed, this is not fully confirmed by literature data.

Plaque length, plaque morphology, ICA angulation, and external or common carotid atheroma have recently been evaluated in an International Carotid Stenting Study (ICSS) substudy and were found not to influence the number of postprocedure DWI lesions in a large cohort of patients [20]. Other studies reported conflicting evidence about plaque morphology $[8,9]$ as well as about the usefulness of distal protection devices [4-7]. Left-side stenosis has been documented to be an increased risk of distal embolization in 2 previous studies [20,21], maybe due to a more difficult access to the common carotid artery on this side. 
Hence, to the best of actual evidence, brain vessel anatomy or technical aspects of endovascular procedures are not enough to completely explain what happens in CAS patients. Even if some of the actual uncertainty may be ascribed to inadequate patient sample or underpowered studies, the evidence of a role of preexisting white matter damage underlined by the present study also opens new interesting perspectives.

The reason why a preexisting brain damage may influence the clinical or subclinical outcome of a reasonably safe procedure, independently of age effect, could be ascribed to several pathogenetic mechanisms.

WMH are demonstrated to be more common and extensive in patients with symptomatic cerebrovascular disease [22]. A cerebral hemodynamic impairment has been found in areas of white matter changes, if compared with areas of normal-appearing white matter [17]. However, a global reduced cerebrovascular reactivity has been correlated with the severity of periventricular WMH [15-23]. Globally impaired cerebral hemodynamics may per se cause hypoperfusion of certain brain areas in the basal condition and a reduced emboli washout during a triggering event [24]. This observation could partially explain why DWI-restricted lesions were found not only on the side of the treated artery, which could be directly related to the migration on a small embolus during the endovascular procedure, but also on the contralateral hemisphere and in posterior vascular territories [25].

Findings from an ICSS substudy showed that CAS was associated with a higher risk of ischemic stroke compared with carotid endarterectomy in patients with more extensive agecorrected white matter lesions on brain MR imaging [26]. Our results are in accordance with those of Elderle et al. [26], extending their significance also to apparently asymptomatic ischemic brain lesions.

According to our results and to ICSS substudy findings, we can speculate that patients with a more extensive brain white matter damage have globally compromised cerebral hemodynamics, and for this reason are more prone to develop new ischemic brain lesions, clinically silent or symptomatic, when their unstable balance is upset by a triggering external event.

Since in the general population the prevalence of WMH is strictly associated with age, ranging from 11 to $21 \%$ in most octogenarians [27-29], we entered the term "age" in the multivariate model, obtaining similar results.

Current guidelines for the prevention of stroke state that CAS is a reasonable intervention alternative to carotid endarterectomy in patients with severe carotid artery diseases [30-32]. However, in certain clinical settings, such as in the elderly, or in case of arterial anatomy inadequate for endovascular intervention, CAS has been related to an increased risk of periprocedural complications (i.e., stroke, myocardial infarction, or death) and to a long-term risk of ipsilateral stroke if compared to carotid endarterectomy [33]. Moreover, its effectiveness compared with actual best medical therapy is still object of investigation [34].

In this complex scenario, the present study outlines a new issue to ponder about for the best patient management.

\section{Study Limitations}

This study has some important limitations. First of all, the small number of patients in the DWI+ group allowed us to assess the effect of only few variables on the final outcome. Moreover, an evaluation of cerebral vasomotor reactivity would have been helpful to test the hypothesis raised from our results; unfortunately, we were not able to perform it in most enrolled patients. Cerebral hemodynamics evaluation should be performed in future similar studies. Finally, our analysis is retrospective and our findings need to be confirmed by a larger prospective clinical study. 


\begin{tabular}{l|l}
\hline Cerebrovasc Dis Extra 2017;7:1-8 \\
\hline DOI: 10.1159/000452717 & $\begin{array}{l}\text { C } 2017 \text { The Author(s). Published by S. Karger AG, Basel } \\
\text { www.karger.com/cee }\end{array}$ \\
\hline
\end{tabular}

Maggio et al.: The Role of White Matter Damage in the Risk of Periprocedural Diffusion-Weighted Lesions after Carotid Artery Stenting

\title{
Disclosure Statement
}

\author{
The authors declare that there are no conflicts of interest.
}

\section{References}

1 Maggio P, Altamura C, Landi D, Migliore S, Lupoi D, Moffa F, et al: Diffusion-weighted lesions after carotid artery stenting are associated with cognitive impairment. J Neurol Sci 2013;328:58-63.

$\longrightarrow 2$ Bendszus M, Stoll G: Silent cerebral ischaemia: hidden fingerprints of invasive medical procedures. Lancet Neurol 2006;5:364-372.

-3 Gröschel K, Ernemann U, Schnaudigel S, Wasser K, Nägele T, Kastrup A: A risk score to predict ischemic lesions after protected carotid artery stenting. J Neurol Sci 2008;273:112-115.

4 Kastrup A, Nägele T, Gröschel K, Schmidt F, Vogler E, Schulz J, et al: Incidence of new brain lesions after carotid stenting with and without cerebral protection. Stroke 2006;37:2312-2316.

5 Cosottini M, Michelassi MC, Puglioli M, Lazzarotti G, Orlandi G, Marconi F, et al: Silent cerebral ischemia detected with diffusion-weighted imaging in patients treated with protected and unprotected carotid artery stenting. Stroke 2005;36:2389-2393.

6 Macdonald S, Evans DH, Griffiths PD, McKevitt FM, Venables GS, Cleveland TJ, et al: Filter-protected versus unprotected carotid artery stenting: a randomised trial. Cerebrovasc Dis 2010;29:282-289.

7 Barbato JE, Dillavou E, Horowitz MB, Jovin TG, Kanal E, David S, et al: A randomized trial of carotid artery stenting with and without cerebral protection. Vasc Surg 2008;47:760-765.

8 Zhou W, Zareie R, Tedesco M, Gholibeikian S, Lane B, Hernandez-Boussard T, et al: Risk factors predictive of carotid artery stenting-associated subclinical microemboli. Int J Angiol 2011;20:25-32.

-9 Stojanov D, Ilic M, Bosnjakovic P, Zivkovic M, Jolic S, Vukasinovic N, et al: New ischemic brain lesions on diffusion-weighted MRI after carotid artery stenting with filter protection: frequency and relationship with plaque morphology. AJNR Am J Neuroradiol 2012;33:708-714.

$\checkmark 10$ Geerlings MI, Appelman AP, Vincken KL, Algra A, Witkamp TD, Mali WP, et al; SMART Study Group: Brain volumes and cerebrovascular lesions on MRI in patients with atherosclerotic disease. The SMART-MR study. Atherosclerosis 2010;210:130-136.

11 Pantoni L, Garcia JH: Pathogenesis of leukoaraiosis. A review. Stroke 1997;28:652-659.

12 Pantoni L: Cerebral small vessel disease: from pathogenesis and clinical characteristics to therapeutic challenges. Lancet Neurol 2010;9:689-701.

13 Hatazawa J, Shimosegawa E, Satoh T, Toyoshima H, Okudera T: Subcortical hypoperfusion associated with asymptomatic white matter lesions on magnetic resonance imaging. Stroke 1997;28:1944-1947.

14 Bakker S, de Leeuw F, de Groot J, Hofman A, Koudstaal P, Breteler M: Cerebral vasomotor reactivity and cerebral white matter lesions in the elderly. Neurology 1999;52:578-583.

15 Isaka Y, Okamoto M, Ashida K, Imaizumi M: Decreased cerebrovascular dilatory capacity in subjects with asymptomatic periventricular hyperintensities. Stroke 1994;25:375-381.

16 Cupini LM, Diomedi M, Placidi F, Silvestrini M, Giacomini P: Cerebrovascular reactivity and subcortical infarctions. Arch Neurol 2001;58:577-581.

17 Marstrand JR, Garde E, Rostrup E, Ring P, Rosenbaum S, Mortensen EL, et al: Cerebral perfusion and cerebrovascular reactivity are reduced in white matter hyperintensities. Stroke 2002;33:972-976.

18 Fu JH, Lu CZ, Hong Z, Dong Q, Luo Y, Wong KS: Extent of white matter lesions is related to acute subcortical infarcts and predicts further stroke risk in patients with first ever ischaemic stroke. J Neurol Neurosurg Psychiatry 2005;76:793-796.

$>19$ Rosset A, Spadola L, Ratib 0: Osirix: an open-source software for navigating in multidimensional DICOM images. J Digit Imaging 2004;17:205-216.

20 Doing D, Hobson BM, Müller M, Jäger HR, Featherstone RL, Brown MM, et al; ICSS-MRI Study Investigators: Carotid anatomy does not predict the risk of new ischaemic brain lesions on diffusion-weighted imaging after carotid artery stenting in the ICSS-MRI substudy. Eur J Vasc Endovasc Surg 2016;51:14-20.

-21 Naggara O, Touzé E, Beyssen B, Trinquart L, Chatellier G, Meder JF, et al; EVA-3S Investigators: Anatomical and technical factors associated with stroke or death during carotid angioplasty and stenting: results from the endarterectomy versus angioplasty in patients with symptomatic severe carotid stenosis (EVA-3S) trial and systematic review. Stroke 2011;42:380-388.

22 Launer LJ: Epidemiology of white matter lesions. Top Magn Reson Imaging 2004;15:365-367.

23 Zupan M, Šabović M, Zaletel M, Popovič KŠ, Žvan B: The presence of cerebral and/or systemic endothelial dysfunction in patients with leukoaraiosis: a case control pilot study. BMC Neurol 2015;15:158.

24 Caplan LR, Hennerici M: Impaired clearance of emboli (washout) is an important link between hypoperfusion, embolism, and ischemic stroke. Arch Neurol 1998;55:1475-1482.

25 Park KY, Chung PW, Kim YB, Moon HS, Suh BC, Yoon WT: Post-interventional microembolism: cortical border zone is a preferential site for ischemia. Cerebrovasc Dis 2011;32:269-275. 


\section{Cerebrovascular \\ Diseases}

Maggio et al.: The Role of White Matter Damage in the Risk of Periprocedural Diffusion-Weighted Lesions after Carotid Artery Stenting

26 Ederle J, Davagnanam I, van der Worp HB, Venables GS, Lyrer PA, Featherstone RL, et al; ICSS investigators: Effect of white-matter lesions on the risk of periprocedural stroke after carotid artery stenting versus endarterectomy in the International Carotid Stenting Study (ICSS): a prespecified analysis of data from a randomised trial. Lancet Neurol 2013;12:866-872.

-27 Ylikoski A, Erkinjuntti T, Raininko R, Sarna S, Sulkava R, Tilvis R: White matter hyperintensities on MRI in the neurologically nondiseased elderly. Analysis of cohorts of consecutive subjects aged 55 to 85 years living at home. Stroke 1995;26:1171-1177.

28 Garde E, Mortensen EL, Krabbe K, Rostrup E, Larsson HB: Relation between age-related decline in intelligence and cerebral white-matter hyperintensities in healthy octogenarians: a longitudinal study. Lancet 2000;356: 628-634.

29 Pasi M, van Uden IW, Tuladhar AM, de Leeuw FE, Pantoni L: White matter microstructural damage on diffusion tensor imaging in cerebral small vessel disease: clinical consequences. Stroke 2016;47:1679-1684.

-30 Meschia JF, Bushnell C, Boden-Albala B, Braun LT, Bravata DM, Chaturvedi S, et al; American Heart Association Stroke Council; Council on Cardiovascular and Stroke Nursing; Council on Clinical Cardiology; Council on Functional Genomics and Translational Biology; Council on Hypertension: Guidelines for the primary prevention of stroke: a statement for healthcare professionals from the American Heart Association/American Stroke Association. Stroke 2014;45:3754-3832.

-31 Brott TG, Howard G, Roubin GS, Meschia JF, Mackey A, Brooks W, et al; CREST Investigators: Long-term results of stenting versus endarterectomy for carotid-artery stenosis. N Engl J Med 2016;374:1021-1031.

-32 Rosenfield K, Matsumura JS, Chaturvedi S, Riles T, Ansel GM, Metzger DC, et al; ACT I Investigators: Randomized trial of stent versus surgery for asymptomatic carotid stenosis. N Engl J Med 2016;374:1011-1020.

-33 Kernan WN, Ovbiagele B, Black HR, Bravata DM, Chimowitz MI, Ezekowitz MD, et al; on behalf of the American Heart Association Stroke Council, Council on Cardiovascular and Stroke Nursing, Council on Clinical Cardiology, and Council on Peripheral Vascular Disease: Guidelines for the prevention of stroke in patients with stroke and transient ischemic attack: a guideline for healthcare professionals from the American Heart Association/American Stroke Association. Stroke 2014;45:2160-2236.

-34 Khandelwal P, Chaturvedi S: Carotid disease management: surgery, stenting, or medication. Curr Cardiol Rep 2015; $17: 625$. 